\title{
Eficacia de un programa de intervención sobre el control del peso en pacientes obesos hipertensos
}

\author{
Luisa Fernández López \\ Lucia Guerrero Llamas \\ Josefina Andugar Hernández \\ M Cruz Casal García \\ Natividad Crespo Tejero \\ Carlos Campo Sien \\ Julián Segura de la Morena \\ (en representación del Grupo \\ EHRICA)
}

Unidad de Hipertensión. Hospital 12 de Octubre.

Madrid

\section{RESUMEN}

La obesidad supone un importante riesgo añadido en la población de pacientes hipertensos, por su prevalencia y por su agregación a otros factores de riesgo.

Nuestro objetivo fue cuantificar las diferencias en la reducción de peso de dos tipos de intervención educacional conducidos por enfermería: intensiva frente a información escrita.

Para ello se diseñó un estudio clínico prospectivo, comparativo entre dos ramas de intervención no farmacológica, en la que se realizan dos tipos distintos de intervenciones que se asignarán de forma aleatoria por el investigador: 1) programa de intervención intensiva de 6 meses de duración con 6 visitas educativas individuales basado en el programa "FORMA" (grupo intervención) y 2) Aporte de información verbal y material escrito, en una única

\section{Correspondencia}

Luisa Fernández López

Hospital 12 de Octubre

Pabellón de Med. Comunitaria. Unidad HTA

Avda. de Córdoba, s/n

28041 Madrid ocasión, para recomendar la perdida de peso, sin que se realicen visitas de seguimiento, hasta los 6 meses (grupo control).

Se reclutaron pacientes, hipertensos esenciales, obesos, no diabéticos y sin complicaciones cardiovasculares, atendidos de forma consecutiva y que cumplían los criterios de selección: edad 18-70 años, IMC: 28$40 \mathrm{~kg} / \mathrm{m}^{2}$. Completaron el estudio 145 pacientes (53.7 \pm 12 años de edad, $58.8 \%$ mujeres), reclutados en 16 centros. El programa de intervención intensiva indujo una reducción promedio de peso significativa frente al grupo $2(3.7 \pm 3.4$ vs. $0.66 \pm 2.9 \mathrm{~kg}$, $\mathrm{p}=0.001$ ). Los factores que influían significativamente en la reducción, según un modelo de regresión lineal múltiple, fueron el sexo (mayor en los hombres) y el centro.

Los niveles de presión al inicio $(146 \pm 17 / 88 \pm 13$ $\mathrm{mmHg}$ ) eran similares en ambos grupos, siendo la reducción de presión mayor en el grupo de intervención intensiva, sin alcanzar la significación $(9.0 \pm 19.5$ vs $4.5 \pm 12.9 \mathrm{mmHg}$ ), aunque en los hombres, la diferencia si fue significativa $(15.1 \pm 19.3$ vs. $6.48 \pm 11.4$ $\mathrm{mmHg}$ ). En el modelo multivariante, la reducción de presión dependía de la reducción de peso obtenida, del sexo y del centro. 
En conclusión, el programa de intervención intensiva resulta más efectivo que la mera información escrita en la reducción de peso. Sin embargo, los diferentes resultados obtenidos según el centro o el sexo de los pacientes, plantea la necesidad de mejorar los programas.

\section{PALABRAS CLAVE: HIPERTENSIÓN \\ OBESIDAD \\ INTERVENCIÓN \\ PROGRAMA FORMA}

\section{EFFICACY OF A WEIGHT CONTROL INTERVEN- TION PROGRAMME IN OBESE HYPERTENSIVE PATIENTS}

\section{ABSTRACT}

Obesity is an important added risk factor in the population of hypertensive patients, because of its prevalence and its aggregation to other risk factors.

Our aim was to quantify the differences in weight loss of two types of nursing-led educational intervention: intensive compared to written information.

For this purpose a prospective clinical study was designed comparing two branches of non-pharmacological intervention, in which two different types of intervention were conducted, which were assigned at random by the researcher: 1) 6-month intensive intervention programme with 6 individual educational visits, based on the "FITNESS" programme (intervention group) and 2) Distribution of verbal information and written material, on a single occasion, to recommend losing weight, but without any follow-up visits until 6 months later (control group).

Essential hypertensive, obese non-diabetic patients without cardiovascular complications were recruited and were attended consecutively, in line with the following selection criteria: age 18-70, BMI: 28-40 $\mathrm{kg} / \mathrm{m} 2$. A total of 145 patients completed the study (aged 53.7 \pm 12 years, 58.8\% women), recruited in 16 centres. The intensive intervention programme led to significant average weight loss compared to group $2(3.7 \pm 3.4$ v. $0.66 \pm 2.9 \mathrm{~kg}, \mathrm{p}=0.001)$. The factors that had a significant influence on the weight loss, according to a multiple linear regression model, were sex (greater in men) and the centre.
Blood pressure levels at the start $(146 \pm 17 / 88 \pm 13$ $\mathrm{mmHg}$ ) were similar in both groups, with the biggest reduction in blood pressure occurring in the intensive intervention group, without achieving a significant level $(9.0 \pm 19.5$ v $4.5 \pm 12.9 \mathrm{mmHg})$, although in men the difference was significant $(15.1 \pm 19.3$ v. $6.48 \pm 11.4$ $\mathrm{mmHg})$. In the multivariant model, the fall in blood pressure depended on the weight loss obtained, the sex and the centre.

In conclusion, the intensive intervention programme is more effective than mere written information for losing weight. However, the different results obtained according to the centre or sex of the patients raise the issue of the need to improve the programmes.

KEY WORDS:

HYPERTENSION

OBESITY

INTERVENTION

FITNESS PROGRAMME

\section{INTRODUCCIÓN}

La obesidad supone un importante riesgo añadido en la población de pacientes hipertensos, por su prevalencia y por su agregación a otros factores de riesgo y sólo el $12 \%$ o $15 \%$ de nuestros pacientes presentan un peso corporal normal. En nuestro país se estima que un 20\% a un 35\% de los hipertensos son, a su vez, obesos. Los pacientes hipertensos obesos están expuestos a mayor riesgo cardiovascular que los delgados y en su mayoría presentan cifras de presión más elevadas. A pesar de ser una importante diana terapéutica, en muchas ocasiones la actuación sanitaria se reduce a un mero consejo o a la aportación de información escrita.

\section{OBJETIVOS}

El objetivo primario de este estudio fue evaluar la influencia sobre la reducción de peso de diferentes tipos de intervención educacional: intensiva frente a información escrita.

Como objetivos secundarios se determinaron:

1. Controlar los cambios en los parámetros más importantes que pueden influir sobre los cambios de peso: dieta y actividad física.

2. Analizar el impacto de los cambios de peso sobre el control de la presión arterial. 
3. Analizar la influencia de la adherencia al tratamiento farmacológico y no farmacológico.

4. Evaluar el porcentaje de aceptación, asistencia y finalización de los distintos programas.

\section{MATERIAL Y MÉTODOS}

Se diseñó un estudio clínico prospectivo, comparativo entre dos ramas de intervención no farmacológica, en el que se realizaron dos tipos distintos de intervención que se asignaron de forma aleatoria por investigador:

1. Programa de intervención intensiva basado en el programa "FORMA", de 6 meses de duración con 6 visitas educativas individuales mensuales, con aporte de material escrito, cuestionarios de calidad de vida y cumplimiento en cada una de ellas (grupo intervención).

2. Programa de 6 meses de duración con aporte de información verbal y material escrito, en una única ocasión, para recomendar la perdida de peso, y cuestionarios de calidad de vida y cumplimiento al inicio y al final, sin que se realicen visitas de seguimiento, hasta los 6 meses (grupo control).

\section{Criterios de inclusión}

- Pacientes obesos con un índice de masa corporal mayor de $28 \mathrm{~kg} / \mathrm{m}^{2}$

- Pacientes con hipertensión arterial esencial en fase estable.

- Con independencia del tratamiento antihipertensivo.

- Ambos sexos, edades entre 18 y 70 años

\section{Criterios de exclusión}

- HTA secundaria, diagnosticada o sospechada

- Pacientes con obesidad mórbida: índice de masa corporal mayor de $40 \mathrm{~kg} / \mathrm{m}^{2}$

- Diabetes mellitus tipo 1 ó tipo 2 con mal control de la glucemia.

- Enfermedades sistémicas (hepatopatia, nefropatía, colagenopatías, etc.)

- Manifiesta falta de cooperación del paciente, adicción a alcohol o a drogas por vía parenteral.

\section{RESULTADOS}

\section{Centros participantes:}

$\mathrm{Al}$ inicio del proyecto participaron 52 investigadoras/es pertenecientes a 30 centros de Atención Primaria y Especializada de toda España.
Los pacientes previstos para su inclusión fueron: 312

- Grupo intervención: 156

- Grupo control: 156

Al final del proyecto los pacientes incluidos fueron 288 de los que 143 no fueron válidos por: 7\% abandonos; $51 \%$ violación de protocolos y $42 \%$ falta de datos. Por lo tanto la muestra final válida fue de 145 pacientes.

\section{CARACTERÍSTICAS INICIALES GLOBALES}

La edad media de los pacientes fue de 53,7 \pm 12 años, de los que el 60\% eran mujeres. El 83,2 de los pacientes eran casados. Presentaban enfermedades cardiovasculares el 5\%; el 66\% tenían antecedentes familiares de hipertensión. La presión arterial sistólica media fue de $143,9 \pm 17 \mathrm{mmHg}$ y la presión arterial diastólica media fue de $87,9 \pm 9,8 \mathrm{mmHg}$ en tratamiento con 1,6 01 fármacos antihipertensivos. El IMC corporal medio fue de $34,3 \pm$ y el peso inicial de $89,9 \pm 14 \mathrm{Kg}$ (tabla 1 ).

\section{Características iniciales globales}

\begin{tabular}{|l|c|}
\hline $\mathbf{N}^{\mathbf{0}}$ Pacientes & 145 \\
\hline Edad & $53,7 \pm 12$ \\
\hline Sexo (Mujeres) & $60 \%$ \\
\hline Est. Civil (casados) & $\mathbf{8 3 . 2 \%}$ \\
\hline Ant. Enf. CV & $5 \%$ \\
\hline Ant.Fam. HTA & $66 \%$ \\
\hline PAS inicial (mmHg) & $143,9 \pm 17$ \\
\hline PAD inicial (mmHg) & $\mathbf{8 7 , 9} \pm 9,8$ \\
\hline Antihipertensivos & $1,6 \pm 1,0$ \\
\hline IMC & $34.3 \pm 4.2$ \\
\hline Peso inicial (kg) & $\mathbf{8 9 , 9} \pm 14$ \\
\hline
\end{tabular}

Tabla 1.

\section{CARACTERÍSTICAS INICIALES SEGÚN GRUPO}

Si analizamos las características iniciales diferenciándolas por grupo, podemos ver en la tabla 1, que no había diferencias significativas entre ambos grupos en los parámetros analizados (tabla 2.)

\section{RESULTADOS DE PESO}

El programa de intervención indujo una reducción promedio de peso significativa frente al grupo control $(3,04$ versus $0,66 \mathrm{Kg})$ tanto en varones $(3,67$ versus $0,97 \mathrm{Kg}$ ) como en mujeres (2,67 versus $0,41 \mathrm{Kg}$ ) (figuras 1 y 2 ). 


\section{Características iniciales según grupo}

\begin{tabular}{|c|c|c|c|}
\hline & INTERVENCIÓN & CONTROL & p \\
\hline N=145 & 78 & 67 & NS \\
\hline Sexo (mujeres) & $\mathbf{5 8 , 7} \%$ & $\mathbf{6 2 , 8} \%$ & NS \\
\hline EDAD & $\mathbf{5 4 , 5} \pm 11,8$ & $53,1 \pm 12,2$ & NS \\
Duración HTA (años) & $\mathbf{6 , 4} \pm \mathbf{6 , 5}$ & $4,9 \pm 5,1$ & NS \\
Antihipertensivos & $\mathbf{1 , 6} \pm \mathbf{1 . 1}$ & $\mathbf{1 , 6} \pm 1,1$ & NS \\
\hline PAS & $149 \pm 20,5$ & $\mathbf{1 4 5 , 9} \pm 17,1$ & NS \\
\hline PAD & $\mathbf{8 9 , 5} \pm 10,6$ & $\mathbf{8 7 , 0 6} \pm 10,8$ & NS \\
\hline IMC & $\mathbf{3 4 , 7} \pm 5,3$ & $\mathbf{3 4 , 0 5} \pm \mathbf{3 , 4}$ & NS \\
\hline
\end{tabular}

Tabla 2.

\section{Resultados PESO}

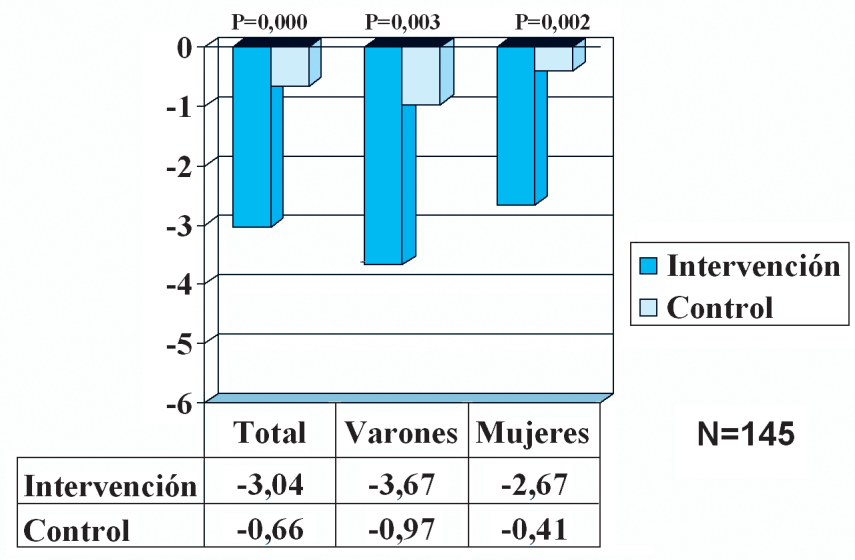

Figura 1.

Si analizamos la reducción de peso según la procedencia de los pacientes del grupo de intervención podemos ver en la figura 2 que los que procedían de hospital tuvieron una reducción de peso significativa frente a los de atención primaria (3,9 versus 2,6 Kg), en los pacientes del grupo control no hubo diferencias. Los factores que influían significativamente en la reducción de peso, según un modelo de regresión lineal múltiple fueron el sexo (mayor en los hombres) y el centro. La diferencia de peso obtenida por la intervención, según un modelo de regresión múltiple, fue independiente del IMC previo, el sexo, la edad y centro.

\section{RESULTADOS PAS Y PAD}

Los niveles de presión, al inicio (146 $\pm 17 / 88 \pm 13$ $\mathrm{mmHg}$ ) eran similares en ambos grupos, siendo la reducción de presión de PAS mayor en el grupo de intervención, sin alcanzar la significación (9.0 $\pm 19,5$ versus 4,74 $\pm 12,9 \mathrm{mmHg}$ ), aunque en los varones, la diferencia si fue significativa $(15,08 \pm 19,3$ versus 6,48 $\pm 11,4 \mathrm{mmHg}$ ) (figuras $3,4,5$ y 6).

\section{Resultados en reducción de peso según centros}

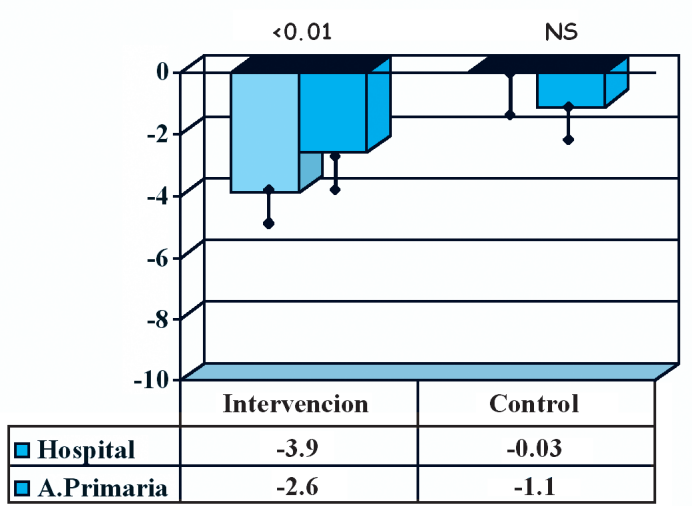

Grupo de intervención

Figura 2.

\section{Resultados PAS}

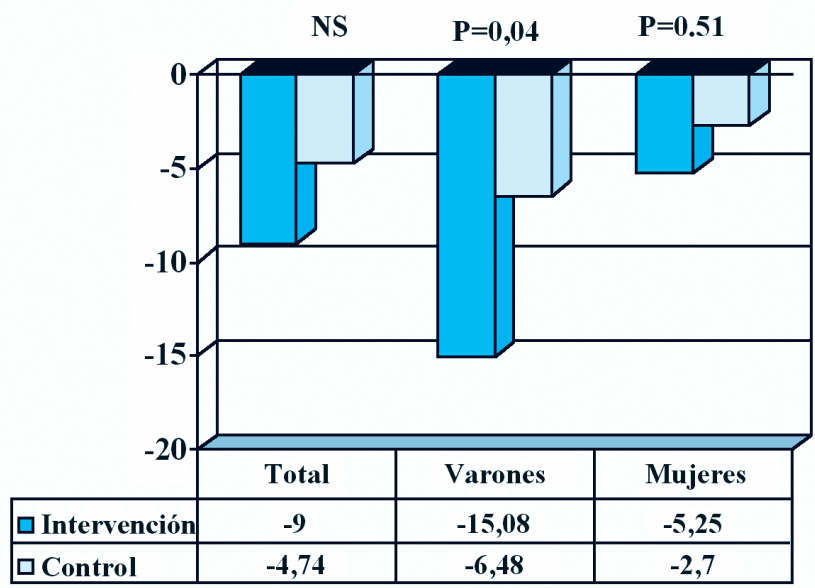

Figura 3.

\section{Resultados PAD}

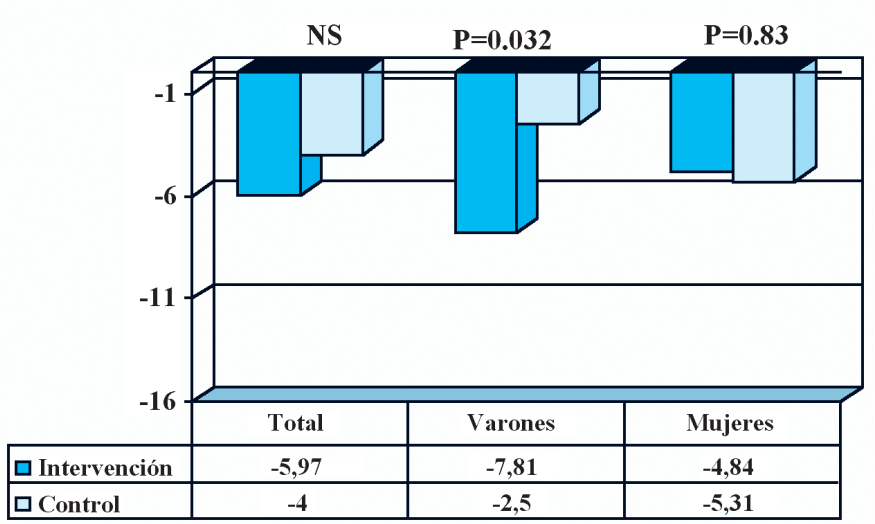

Figura 4. 


\section{Resultados por centros}

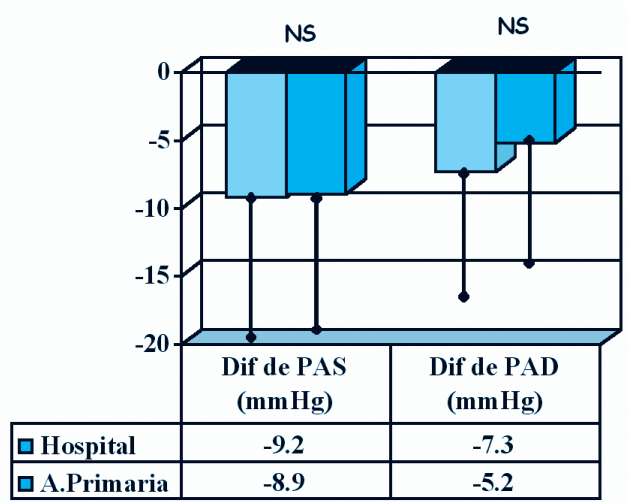

Grupo de intervención

Figura 5

\section{Resultados por centros}

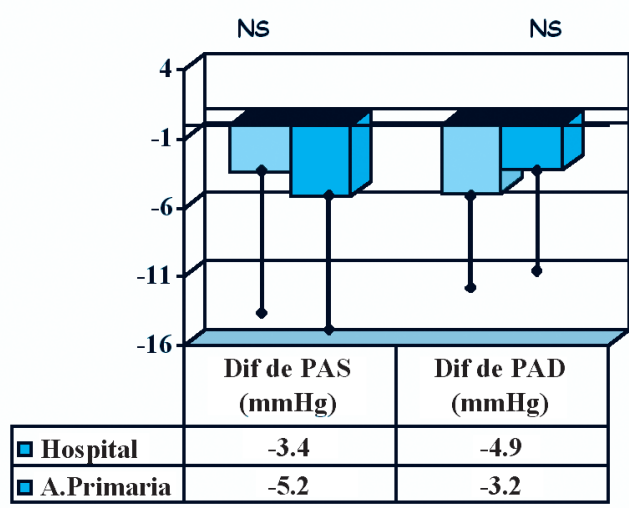

Grupo control

Figura 6.

Las diferencias de PAS y PAD de ambos grupos, sí analizamos a los pacientes según el centro de donde procedían, no fueron significativas en ambos grupos. En el modelo multivariante, la reducción de presión arterial sistólica dependía de la reducción de peso obtenida, del investigador y de la presión arterial sistólica al inicio (tabla 3).

\begin{tabular}{|l|l|l|}
\hline & $\beta$ & $p$ \\
\hline Reducción de peso & 1,4 & 0,05 \\
\hline Investigador & 2,2 & 0,04 \\
\hline PAS inicio & 0,5 & 0,00 \\
\hline
\end{tabular}

Tabla 3. Reducción de PAS. Análisis de regresión multiple.

\section{RESULTADOS CUESTIONARIOS CALIDAD DE VIDA Y CUMPLIMIENTO}

La calidad de vida mejora en los pacientes del grupo de intervención pero sin diferencias significativas respecto al grupo control (tabla 4). Con respecto al cumplimiento farmacológico no hubo diferencias debido a que el cumplimiento inicial era muy bueno (tabla 5). Las causas más frecuentes del no cumplimiento farmacológico fueron: olvido (17\%); aparición de efectos secundarios $(15,3 \%)$ y estar ocupados en otros problemas $(15,2 \%)$ (tabla 6).

\begin{tabular}{|l|l|l|}
\hline & PRE & POST \\
\hline Control & 38,8 & 35,9 \\
\hline Intervención & 38,4 & 33,8 \\
\hline P & NS & NS \\
\hline
\end{tabular}

Tabla 4. Calidad de vida.

\begin{tabular}{|l|c|c|}
\hline \multicolumn{3}{|c|}{ Cumplimiento farmacológico (\%) } \\
\hline PRE & POST & \\
\hline Control & 98,2 & 98,2 \\
\hline Intervención & 95,4 & 100 \\
\hline P & NS & NS \\
\hline
\end{tabular}

Tabla 5.

\section{No Cumplimiento farmacológico}

\begin{tabular}{lc} 
CAUSA & PORCENTAJE (\%) \\
\hline Olvido & 17,2 \\
\hline Aparición efectos secundarios & 15,3 \\
\hline Estar ocupados en otros problemas & 15,2 \\
\hline Poca confianza en los resultados del médico & 9,7 \\
\hline $\begin{array}{l}\text { Olvido de las instrucciones del médico } \\
\text { Dificultad para comprender las instrucciones } \\
\text { del médico }\end{array}$ & 9 \\
\hline
\end{tabular}

Tabla 6.

No hubo cambios significativos en el nivel del cumplimiento higiénico-dietético aunque se observó cierto descenso en el consumo de algunos alimentos en los pacientes del grupo de intervención (tabla 7). 


\begin{tabular}{|l|c|c|}
\hline \multicolumn{3}{|c|}{$\begin{array}{c}\text { Cumplimiento higiénico-dietético } \\
\text { Hábitos de alimentación }\end{array}$} \\
\hline Aceitunas & PRE & POST \\
\hline Embutidos & 1,00 & 0,68 \\
\hline Pescados salados & 1,19 & 0,82 \\
\hline Mantequilla o margarina & 0,35 & 0,17 \\
\hline Carne & 0,68 & 0,43 \\
\hline Tortilla de patatas & 1,71 & 1,35 \\
\hline Dulces, bollos, chocolate & 0,90 & 0,70 \\
\hline Productos enlatados & 0,98 & 0,54 \\
\hline
\end{tabular}

Tabla 7.

Las causa más frecuentes de que los pacientes no cumplieran con las medidas higiénico-dietéticas fueron: mantener relaciones sociales (38.2\%); realizar otras tareas (leer, ver TV, etc.) (34\%); pereza (33.1\%); no sentirse capaz de realizarlo (32.4\%) (tabla 8)

\begin{tabular}{|l|c|}
\hline \multicolumn{2}{|c|}{ No Cumplimiento higiénico-dietético } \\
CAUSA & PORCENTAJE (\%) \\
\hline Mantener relaciones sociales & 38,2 \\
\hline Realizar otras tareas (leer, ver TV, etc) & 34 \\
\hline Pereza & 33,1 \\
\hline No sentirse capaz de realizarlo & 32,4 \\
\hline Olvido o descuido & 27,6 \\
\hline Falta de apoyo familiar & 26,2 \\
\hline Ausencia de síntomas & 22,8 \\
\hline Suficiente con tomar pastillas & 17,9 \\
\hline Poca confianza en los resultados & 15,9 \\
\hline Falta de información & 11 \\
\hline
\end{tabular}

Tabla 8.

\section{DISCUSIÓN Y CONCLUSIONES}

La reducción de peso es uno de los factores más importantes del tratamiento de la hipertensión arterial ya que diferentes estudios han demostrado que la obesidad es uno de los factores de riesgo que tiene una relación más importante en la prevalencia de la hipertensión.

Una pérdida de $5 \mathrm{Kg}$, aunque no se alcance el peso ideal repercute de forma importante en el control de la tensión arterial y mejora la respuesta del tratamiento farmacoló- gico. En relación a la presión arterial, por cada kilo que se pierda, de media, se produce un descenso de $3 \mathrm{mmHg}$ en la sistólica, y $2 \mathrm{mmHg}$ en la diastólica, es decir, que si se pierden $5 \mathrm{~kg}$, se puede reducir la tensión de media: 15 $\mathrm{mmHg}$ en la sistólica y $10 \mathrm{mmHg}$ en la diastólica. El programa de intervención permitió obtener una reducción de peso significativa respecto al grupo control, que fue independiente de otros factores. Esta reducción de peso se asoció con una disminución de la PAS/PAD en varones que también dependía del investigador y de la PA inicial del paciente. La calidad de vida en los pacientes del grupo de intervención mejora pero sin diferencias significativas con respecto a los pacientes del grupo control.

No hubo cambios ni en el cumplimiento farmacológico ni en el higiénico-dietético aunque en este último se observó un descenso de consumo de ciertos alimentos en los pacientes del grupo de intervención.

\section{CONCLUSIONES FINALES}

- Las modificaciones en el estilo de vida son eficaces para disminuir la presión arterial aunque se requiere un refuerzo constante para conseguir y mantener estos cambios en el estilo de vida.

- La constancia de nuestra actuación puede ser recompensada por la reducción de múltiples factores de riesgo cardiovascular.

\section{BIBLIOGRAFÍA}

1. Programa Forma avalado por la Sociedad Española de Endocrinología y Nutrición. SEEN 2002.

2. Sociedad Española de Hipertensión/Liga Española para la Lucha contra la Hipertensión Arterial SEHLEHLA. Guía española de Hipertensión Arterial 2005. Hipertensión 2005; Supl 2: 44-6.

3. Sociedad Española de Hipertensión/Liga Española para la Lucha contra la Hipertensión Arterial SEHLEHLA. Guía sobre el diagnostico y el tratamiento de la hipertensión arterial en España 2002. Hipertensión 2002; 19 Suppl 3:30-3.

4. VI Informe del Joint Nacional Comité. Prevención, detección, evaluación y tratamiento de la Hipertensión arterial. Modificaciones en el estilo de vida. 1998. Cap. 3: 22-26.

5. Lombera Romero F, Barrios Alonso V, Soria Arcos F, Placer peralta JM, Cruz Fernández JM et al. Guías de práctica clínica de la Sociedad Española de cardiología en hipertensión arterial. Revista Española de Cardiología 2000; 53:66-90. 